
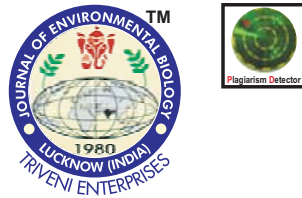

\title{
Impact of protozoan Vahlkampfia sp. on the growth of algae Chlorella vulgaris glamtr
}

Authors Info

\section{N. Wahi' ${ }^{1 *}$ A.K. Bhatia ${ }^{1}$ and S. Bhadauria ${ }^{2}$}

'Department of Biotechnology, GLA University, Mathura-281 406, India

${ }^{2}$ Department of Botany, RBS College, Dr. B.R. Ambedkar University, Agra-282 002, India

*Corresponding Author Email : wahink@gmail.com

Key words

Chlorella vulgaris

ITS-1 \& ITS-4 primers

Photobioreactor

Vahlkampfia sp.

Publication Info

Paper received : 02.06.2017

Revised received : 16.06 .2017

Re-revised received : 15.07.2017

Accepted : 16.08 .2017

\section{Abstract}

Aim : Maintenance of high-density microalgal culture forms the basic feature of algal biofuel technology. Algal biomass cultures explore few microalgal species over the other exposing these to grazers, pathogens and predators. So there is an urgent need to explore technologies that will permit early detection and control of these parasites. The present study reports a predatory microbe which hampered Chlorella vulgaris glamtr (Accession no. KX363808.1) biomass productivity.

Methodology : Growth conditions for alga Chlorella vulgaris glamtr were optimized by varying temperature $\left(24-25^{\circ} \mathrm{C} ; 27-28^{\circ} \mathrm{C} ; 31-\right.$ $\left.32^{\circ} \mathrm{C}\right)$, pH (6.4-6.8; 6.8-7.2; 7.2-7.6), light intensity $\left(1900 ; 2400 ; 2700\right.$ lux) and $\mathrm{CO}_{2}$ concentration (1-5\%). Growth kinetics was recorded at $750 \mathrm{~nm}$. Algal biomass was cultivated in RW 1-170P-photobioreactor system and aquarium. The purity of culture was assessed by microscopic examination at 45X. Contaminating protozoan was identified using universal primer (ITS-1 \& ITS-4) based amplification of $18 \mathrm{~S}$ rRNA sequence and its subsequent phylogenetic analysis using MEGA7.

Results: Chlorella exhibited optimum growth at $27-28^{\circ} \mathrm{C}$, pH 6.8-7.2, light intensity 2700 lux at $5 \% \mathrm{CO}_{2}$ concentration. Microscopic examination revealed predatory nature of contaminating protozoan. ITS-based PCR amplification and subsequent phylogenetic analysis revealed the protozoan to be Vahlkampfia sp. Effective subculturing and prevention of protozoan contamination was found to enhance biomass production by $42 \%$.

Interpretation : The study reveals that the protozoan Vahlkampfia sp. is a potential predator of microalgae. Prevention of amoebic contaminant and maintenance of optimum
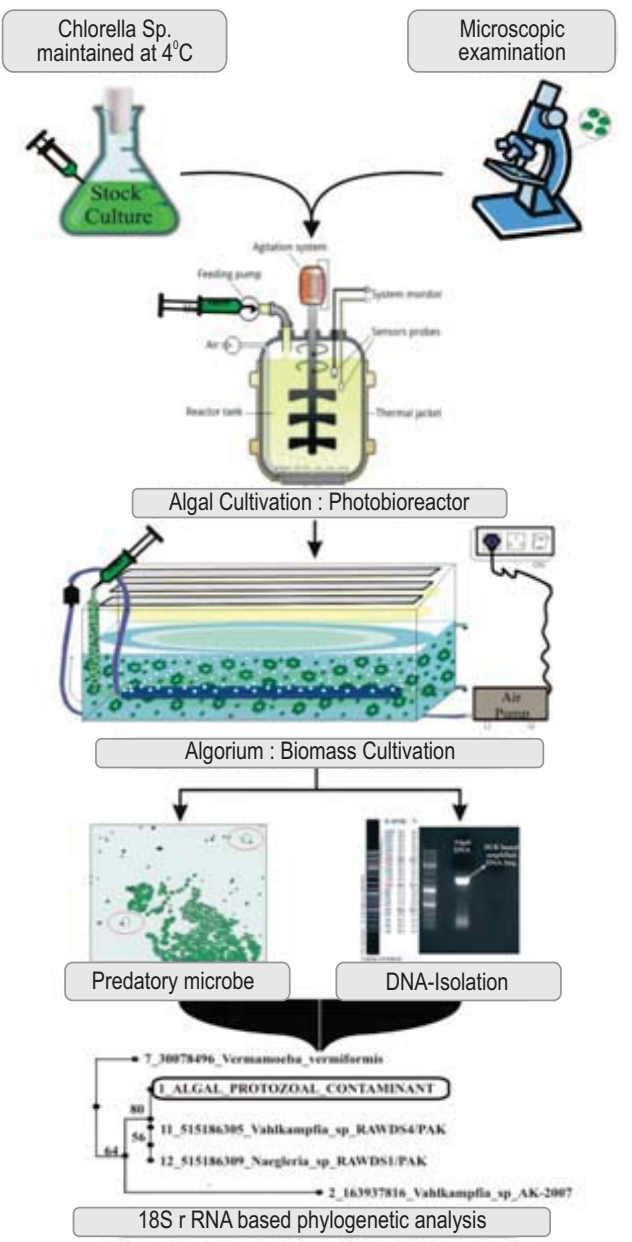
environmental conditions could enhance biomass productivity, facilitating algal cultivation. 


\section{Introduction}

Algal biomass productivity has been limited by grazers, pathogens and predatory species (Day et al., 2012; Carney and Lane, 2014; Chiu et al., 2016). Predatory microbes have been the rate limiting factor in algal growth (Gong et al., 2015). Biomass productivity has been hampered by directly acting contaminants affecting biomass through predation (Day et al., 2012; Chiu et al., 2016) or indirectly acting ones, influencing media composition (Panova and Ivanova, 2002; Mendes and Vermelho, 2013). Algal biomass cultivation in open pond or closed photobioreactor involves the growth of selected species under high density making it susceptible to predation (Gachon etal., 2009; Day etal., 2012).

Fungal, bacterial and amoebic species have been identified since long as potential challenges for algal farming (Mendes and Vermelho, 2013; Carney and Lane, 2014). Fungi and fungal like organism (oomycetes, labyrinthulids) are well known algal parasites (Carney and Lane, 2014). The impact of fungal infection has been observed in open pond cultivation of Scenedesmus (Letcher et al., 2013). Aphelids, a taxon closely related to chytrids, are known intracellular parasites of algal cells (Karpov et al., 2013). Oomycetes also known as water moulds have been identified to cause 10 to $60 \%$ loss in seaweed productivity (Gachon et al., 2009).

Bacterial contamination of algal cultures has been found to be limited but can cause variations in media composition affecting algal growth and biomass productivity (Gachon et al., 2009; Mendes and Vermelho, 2013). Amoebic predation over algal species has been previously reported (Ho and Alexander, 1974; Becares and Romo, 1994; Gong et al., 2015). Filos (Nuclearia), a lobose amoeba has been reported to feed on blue green algae (Anabena, Nostoc, Osillatoria, Tolypothrix) by engulfing vegetative cells, heterocyst and akinete stage (Becares and Romo, 1994). Amoebic predation is not only limited to cyanobacteria (Ma et al., 2017) but is also a threat to microalgal cultivation (Berney et al., 2013). A decrease in biomass cultivation by amoebic predation has been demonstrated in the open raceway pond and photobioreactor studies on Scenedesmus sp. (Gong et al., 2015). Antimicrobial drug resistance, ability to thrive under extreme environmental conditions have evolved these protozoans as an emergent threat to commercial algal biomass industry.

Keeping in view of the above, in the present study algal biomass culture was investigated in both open and closed photobioreactor for the presence of any predatory microorganism hampering Chlorella vulgaris glamtr (Accession no. 363808.1) growth and productivity.

\section{Materials and Methods}

Characterization and optimization of culture condition : The algal strain Chlorella vulgaris glamtr was isolated from Ajhai Kund, a local water body situated near the GLA University at NH2,
Mathura, India. The collected samples after serial dilution, repeated subculturing and isolation of microalgal species were inoculated over CHU-10, BG-11, Spirulina and BB media in 250 $\mathrm{ml}$ emersion flask. Experiments were performed by varying incubation temperature $\left(24-25^{\circ} \mathrm{C} ; 27-28^{\circ} \mathrm{C}\right.$ and $\left.31-32^{\circ} \mathrm{C}\right)$, incubation $\mathrm{pH}(6.4-6.8 ; 6.8-7.2$ and 7.2-7.6) and light intensity (1900 Lux, 2400 Lux and 2700 Lux) to determine optimum conditions for microalgae cultivation.

The medium over which the algal colonies firstly appeared was selected for further experiments. A cool fluorescent light was used to illuminate the cultures at different light intensities which were measured with the help of Lux meter. The experiments, by default, were repeated three times, under optimum conditions of temperature, $\mathrm{pH}$ and light intensity with 1 $5 \% \mathrm{CO}_{2}$ supply (Pipes, 1962).

Growth kinetics of the algal species : The selected microalga was cultured under optimized growth condition within broth cultures of $150 \mathrm{ml}$ each (in triplet). Once the purity of culture maintained was established, the primary inoculum was prepared by incubating it for 7-8 days until $0.6 \mathrm{OD}$ was recorded at $750 \mathrm{~nm}$. The primary inoculum was used in scaling up reaction to obtain microalga biomass by cultivating it within the algorium (an aquarium modified into an Open photobioreactor) and RW 1170P-photobioreactor system (closed photobioreactor) under optimized conditions. Growth curve analysis was made by determining the optical density (OD) of the microalgal culture at regular time interval. Optical density was measured at $750 \mathrm{~nm}$ through Lab India, UV spectrophotometer. The experiment was repeated three times to minimize any error.

Microscopic examination of predatory protozoan : The purity of the microalgal culture was investigated by repeated microscopic examination under Mitzer bright field microscope at 45X and 100X magnification. Luria-Bertani media was used to check the presence of any bacterial contamination. Random sampling and repetitive microscopic examination were carried out to identify if the protozoan contaminant was unique or was present as a mixture of more than one form. Microscopic examination was also carried out to gain an insight about the contaminating amoebic strain if it was present in flagellated or amoeboid form.

Molecular identification of the predatory protozoan : Predatory protozoan was identified through phylogenetic analysis of the conserved 18S rRNA encoding sequence. The $18 \mathrm{~S}$ rRNA sequence was amplified from the nuclear DNA with the help of universal primers (ITS-1 and ITS-4). (Fig. 1) (Table1) (Ristaino et al., 1998). The use of ITS primers allowed bidirectional amplification of conserved region.

Genomic DNA was isolated using Insta Gene TM Matrix Genomic DNA isolation kit (Cat. No. 732-6030), Bio-Rad. Isolated DNA was subjected to PCR amplification with initial denaturation 
Table 1 : Primers employed during the PCR-based amplification of consensus 18 S rRNAencoding sequence

\begin{tabular}{lll}
\hline Primers & Type & Primer Sequence \\
\hline ITS-1 & Forward & 5-TCCGTAGGTGAACCTGCGG-3』 \\
ITS-4 & Reverse & 5 5-TCCTCCGCCTTATTGATATCC-3』 \\
\hline
\end{tabular}

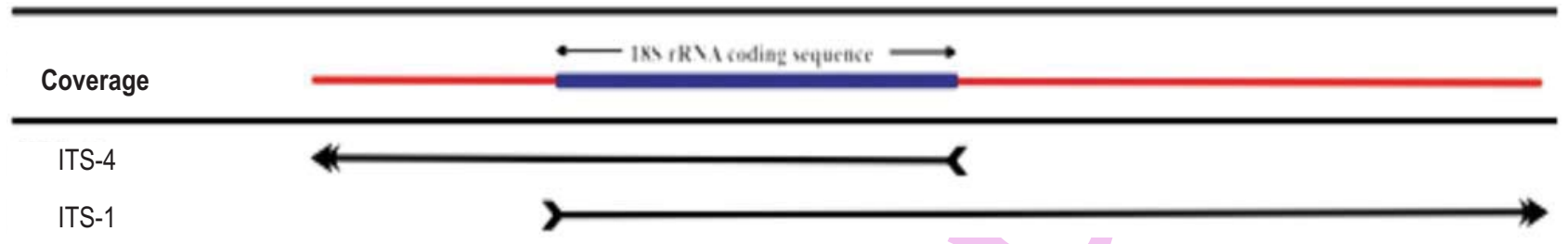

Fig. 1 : Specific binding sites of universal primers (ITS-1 and ITS-4) used for the amplification of conserved 18S rRNA encoded sequence from the genomic DNA of a predatory protozoan

at $94^{\circ} \mathrm{C}$ for $2 \mathrm{~min}$, annealing step at $55^{\circ} \mathrm{C}$ for $60 \mathrm{sec}$ and extension step at $72^{\circ} \mathrm{C}$. Removal of unincorporated nucleotides and primers from the PCR product was achieved using Montage PCR cleanup kit (Millipore) and analyzed through $0.8 \%$ agarose gel electrophoresis. The PCR products were subsequently sequenced using $\mathrm{ABI}$ PRISM Big Dye TM Terminator Cycle Sequencing Kits with Ampli Taq DNA Polymerase (Applied Biosystems). The experiment was repeated three times to minimize the chances of non specific amplification of the target DNA sequence by inappropriate binding of ITS primers.

The evolutionary history was inferred using the neighborjoining method (Saitou and Nei, 1987). The bootstrap consensus tree inferred from 500 replicates was taken to represent the evolutionary history of the taxa analyzed (Felsenstein, 1985).

-Growth Curve of Alegae within Photobioreactor (Without Contaminanat)

(A) -Growth Curve of Alegae within Algorium (With Protozoal Contaminanat)

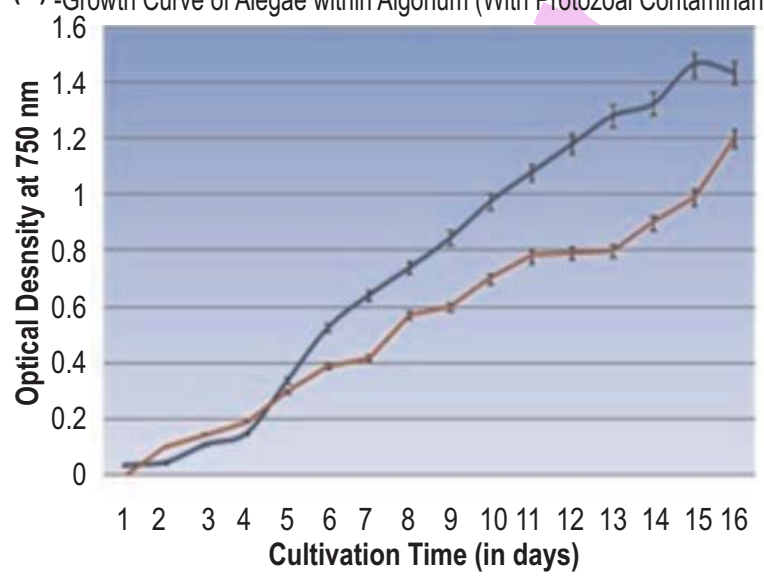

Branches corresponding to partitions reproduced in less than $50 \%$ bootstrap replicates were collapsed. The evolutionary distance was computed using maximum composite likelihood method. The analysis involved 12 nucleotide sequences. All positions containing gaps and missing data were eliminated. A total of 254 positions were found in the final dataset. The evolutionary analysis was conducted using MEGA 7 (Fig. 4). Growth analysis of culture was further carried out by inoculating it under optimum growth conditions preventing any protozoan contamination.

\section{Results and Discussion}

As the colonies of algae Chlorella vulgaris glamtr firstly appeared over BB-media, therefore, it was selected for further experiments. The optimum cultural condition determined were 27-

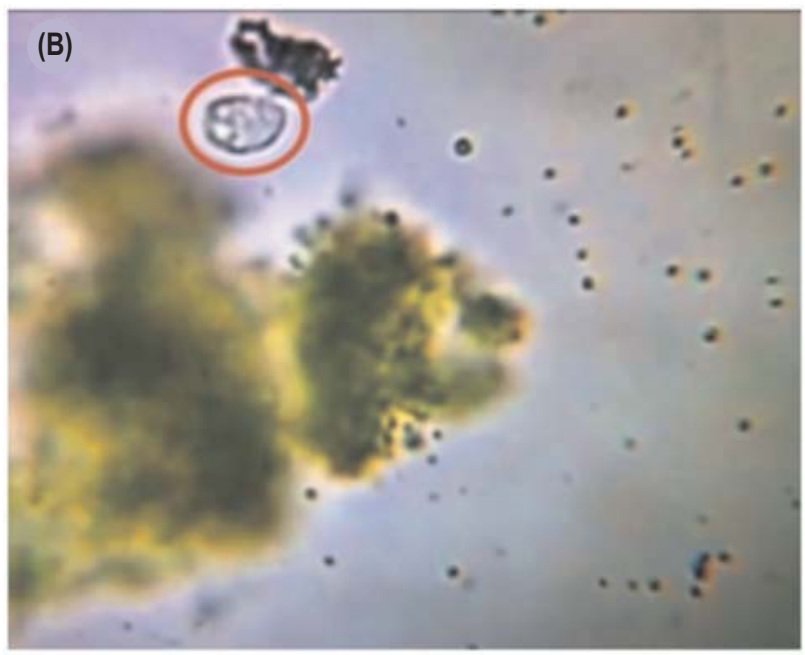

Fig. 2 : (A) Comparative analysis of the growth curves for Chlorella vulgaris glamtr in the presence and absence of Vahlkampfia sp. contaminant at optical density of $750 \mathrm{~nm}$; (B) Protozoan contaminant Vahlkampfia sp. (MF187528) feeding over the algal biomass 
$28^{\circ} \mathrm{C}$; pH 6.8-7.2 and 2700 Lux light intensity was in accordance with the previous studies (Imaizumi et al., 2014; Blinova et al., 2015). Experiments related to $\mathrm{CO}_{2}$ supply within the growth medium revealed $5 \% \mathrm{CO}_{2}$ promoted algal growth, but only when optimum conditions were maintained (Han et al., 2013; Scherholz and Curtis, 2013; Zhang et al., 2016). As algal cultures achieved higher density, $\mathrm{CO}_{2}$ becomes rate limiting so air supplemented with $\mathrm{CO}_{2}$ was found to boost algal growth (Mahan et al., 2005; Blinova et al., 2015). Addition of $\mathrm{CO}_{2}$ further boosted $\mathrm{pH}$ maintenance by establishing carbonic acid and bicarbonate ion balance.
Microalgae culture was cultivated under optimum growth conditions with a pH regulated $\mathrm{CO}_{2}$ supply system, within $\mathrm{RW} 1$ 170P-photobioreactore system and algorium using $10 \mathrm{ml}$ of the inoculum at $0.6 \mathrm{OD}$. The growth kinetics of algae was determined by regularly monitoring $\mathrm{OD}$ of samples at $750 \mathrm{~nm}$. Algal inoculum displayed almost similar growth rates till $5^{\text {th }}$ day of incubation, beyond which a difference in growth rates was noted, which was further enhanced during the terminal log phase of growth (11-15 day) (Fig 2. A). Microalgae growth rate was found to be profoundly larger within the photobioreactor as compared to algorium.
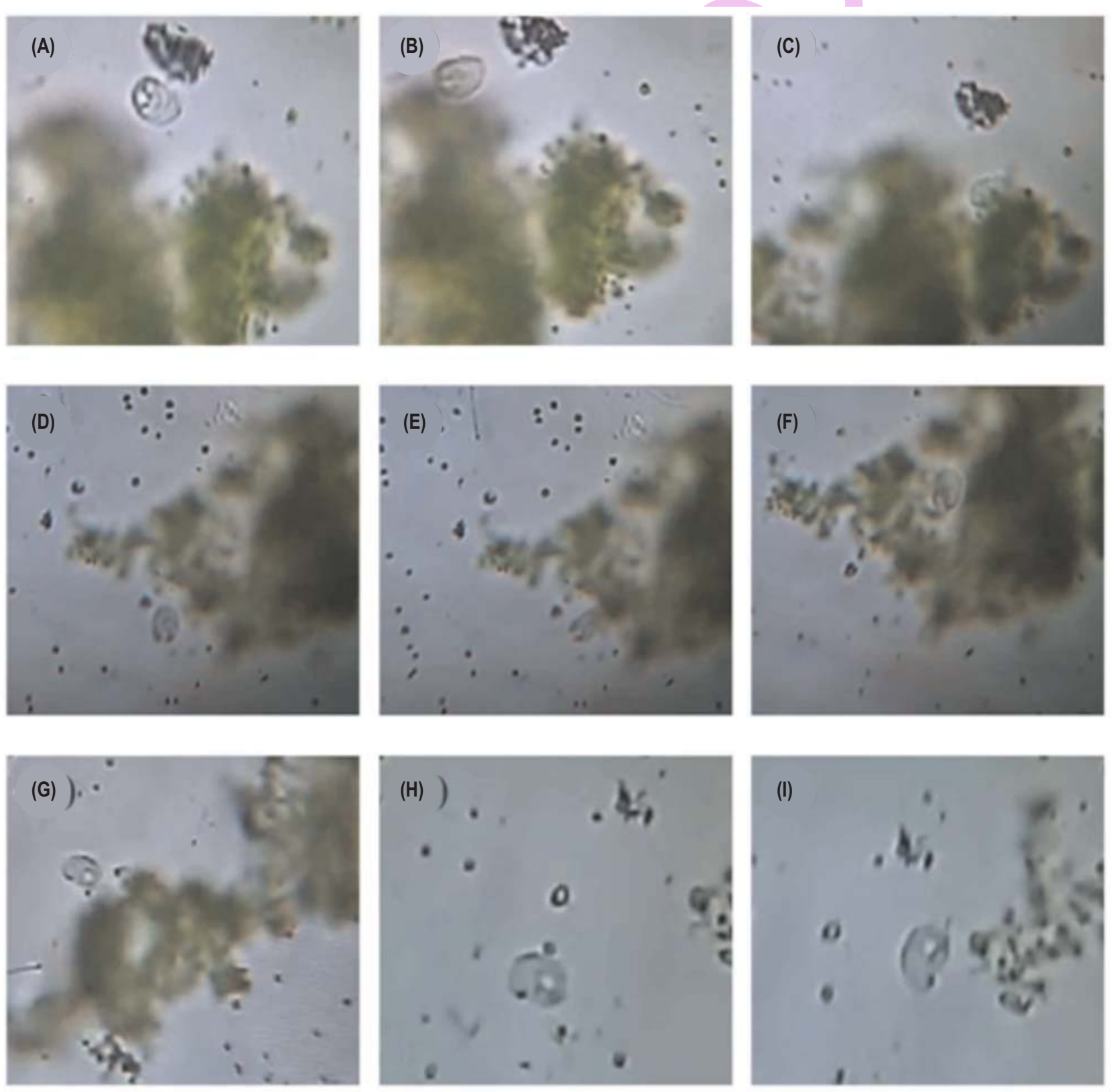

Fig. 3 : Microscopic examination of algal culture contaminated with a protozoan. (A-C) Sequence of events depicting the predatory activity of protozoan strain over the alga Chlorella vulgaris glamtr; (D-G) Motion of protozoan towards the green alga for feeding and finally away from it and (H-I) Presence of microalgal cells within the predatory protozoan 


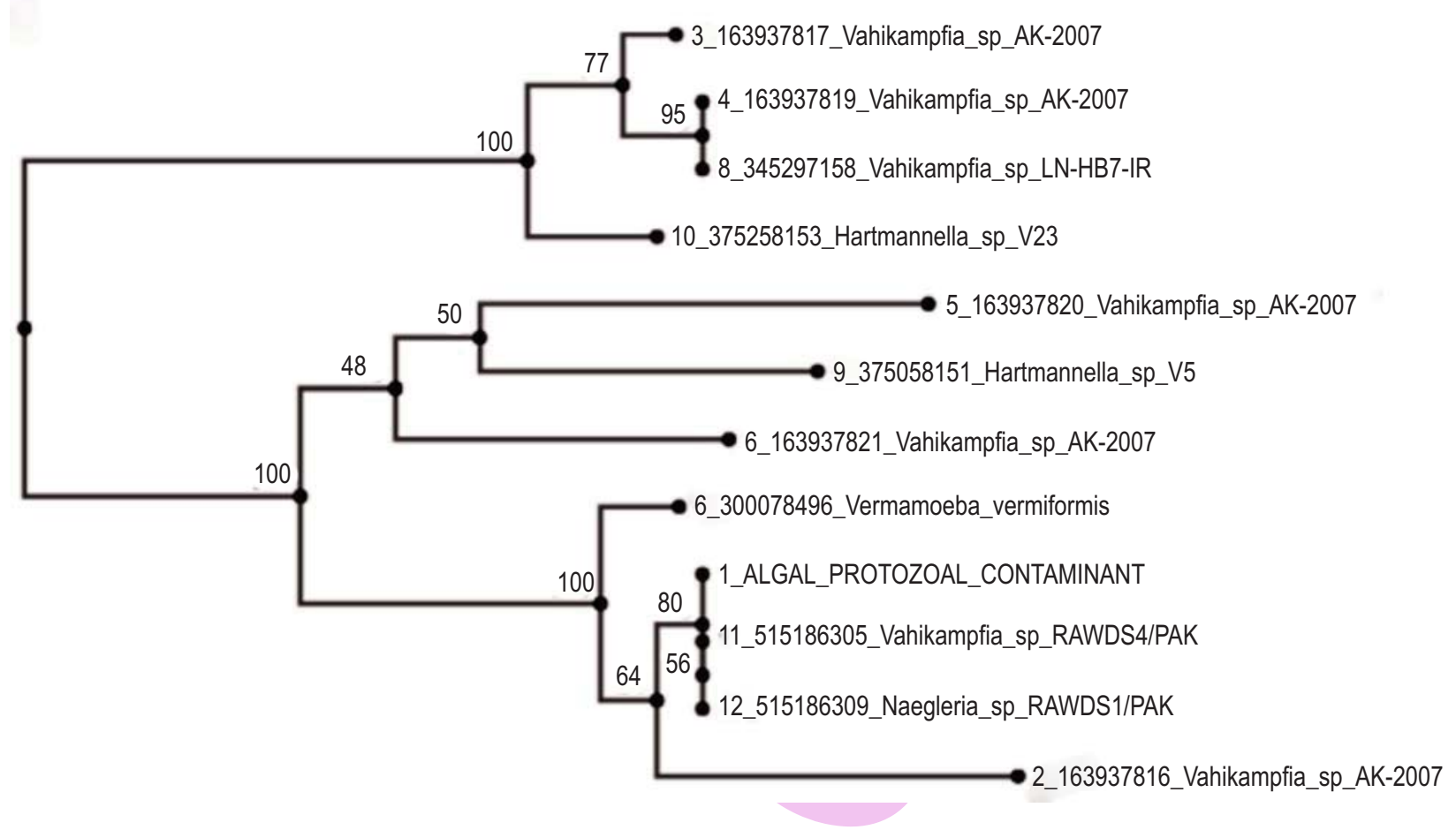

Fig. 4 : Phylogenetic maximum likelihood tree based on 18S rRNA encoding sequence depicting the relationship of isolated ALGAL PROTOZOAL CONTAMINANT with reference strains from NCBI database. Species names are followed by their GenBank accession number. The number shown next to each bifurcation is the bootstraps percent value based on 500 replications

To investigate the cause of such variations in growth rate algal cells were harvested from both open (Algorium) and closed photobioreactor system by centrifugation at $3800 \mathrm{xg}$ for $20 \mathrm{~min}$ at $4^{\circ} \mathrm{C}$. The supernatant was investigated for the presence of any microbial contaminant. Fungal contamination was found to be limited to solid culture media containing a carbon source as it is essentially required for fungal growth. Properly sterilized broth media displayed almost no fungal contaminants during autotrophic growth which was in accordance with the study of Mahan et al. (2005). Fungal contaminants were found to be a challenge in the heterotrophic cultivation of Chlorella sp., as the process required a carbon source, being uneconomical was not studied any further.

Microscopic examination of supernatant samples at 45X (Fig. 2) revealed that protozoan contaminant was found to be associated with microalgal cultures from Algorium. Almost no protozoan contaminant was found to be associated with photobioreactor biomass. Sequential microscopic examination and image analysis clearly revealed the predatory nature of protozoan contaminant (Fig. 3. A-I). Some of the captured images even displayed the presence of microalgal cells within the protozoan parasite (Fig. 3. H-I). Similar results have been obtained in the study of Ma et al. (2017) who found the predatory effect of Heterolobosean amoeba LPG3, on unicellular Cyanobacteria strain, while Mendez and Uribe, 2012 have highlighted the feeding ability of Branchionis sp.,
Amoebic sp. on cyanobacteria Arthrospira sp which is in accordance with the results obtained.

Microscopic examination of protozoan parasite predicted its cylindrical structural with size varying from 20-40 $\mu \mathrm{m}$. Although no pseudopodia formation was observed, the amoebic predator displayed a hemispherical bulge towards its front margin, which was in accordance with the study of Smirov and Fenchel (1996) and Robles et al. (2012) on Vahlkampfia sp. and Vannella sp.

Isolated DNA from protozoan contaminant was subjected to ITS1 and ITS4 primers based PCR amplification, of 18S rRNA encoding genes and its subsequent phylogenetic analysis using MEGA 7. Phylogenetic analysis revealed it to be closely related to Vahlkampfia sp. (Accession no. MF187528) (Fig. 4) (Jonckheere, 2006). Although effective in nature PCR based assays have been limited to laboratory scale bioreactor system and have not been explored in open pond cultivation system for detection of microalgae predators.

Predatory microorganisms, especially amoebic strains have been reported since long to feed upon algal biomass (Becares and Romo, 1994; Gong et al., 2015). Amoebic strain was characterized to be Vahlkampfia sp. on the basis of morphological and molecular analysis. Almost no documentary literature is available over the predatory activity of amoeba Vahlkampfia sp. It belongs to the heterolobosa group of kingdom protista but unlike 
other amoebae, Vahlkampfia lacks a flagellated stage and resides solely in an amoeboid form (Robles etal., 2012).

Classical algologists have suggested optimization of cultural conditions to overpower the damage caused by grazing predatory microbes (Richmond and Becker, 1986). The success of this strategy depends on the extent of grazing. An active grazer could deplete the entire algal biomass within two to three days and decrease biomass productivity, so antiamoebic and antiprotozoan agents (Sodium hypochlorite) have been used since long (Mendez and Uribe, 2012). Ammonium hydroxide at a concentration of $17 \mathrm{mg} \mathrm{l}^{-1}$ has been employed for checking the contaminant Brachionus rubens (Lincoln et al., 1983). Similarly, urea, thiourea and ammonium bicarbonate at various concentrations have been found useful in limiting contaminating Amoebic sp. (Duerr et al., 1997; Ibrahim et al., 2014). The use of antiamoebic drugs could effectively eliminate protozoan contaminant but would increase the cost of large scale algal cultivation so have not been employed at large as has been concluded in the study conducted by Mendez and Uribe (2012). So, UV light illumination, minimum use of antiamoebic drugs (tetracycline, puromycin and diiodohydroxyquin) at basal concentration and careful sub culturing were used to obtain uncontaminated cultivars of Chlorella sp.

Sub culturing was performed under aseptic conditions, and culture was inoculated within closed photobioreactor (RW 1170P-Photobioreactor) to determine the altered growth rate. Cultivation within the closed photobioreactor was repeated three times to establish growth curve in the absence of protozoan contaminant. The biomass productivity was found to be enhanced by $40-42 \%$ in the absence of any contaminating strain (1.42 OD as compared to $1 \mathrm{OD}$ at $15^{\text {th }}$ day of inoculation) as compared to a contaminated culture (Fig. 2A). This clearly illustrates the deleterious effect of grazing predatory microbes over algal biomass cultivation.

Optimization of the cultural condition and use of specific antiamoebic, antimicrobial and antifungal agent could effectively eliminate protozoan contaminants enhancing microalgal productivity. Highest productivity could only be made possible if algal cultures are effectively being protected from predatory micro-organisms with the minimal use of antimicrobial agents.

\section{Acknowledgments}

We are thankful to Prof. DS Chauhan, VC, GLA University and to Prof. Anirudh Pradhan, Dean, R\&D, GLA University for their support in initiating algal based research work in University campus. Authors are indebted to Yaazh Xenomics Lab, Madurai, India for carrying out DNA sequencing and to Dr Rajiv Aneja, Director, ABCA BioSolutions, Chandigarh, India for his kindheartedness allowing the use of RW 1-170P-photobioreactor system during scaling up experiments. The work was funded by the University Grant
Commission, Grant no. 2-13/ 2011 (SA-I), Ministry of Education and Research, New Delhi, India.

\section{References}

Becares, E. and S. Romo: Selective predation of Thecamoeba sphaeronucleolus (Greeff, 1891) on filamentous algae in natural conditions. J. Gen. Appl. Microbiol., 40, 15-21 (1994).

Berney, C., S. Romac, F. Mahe, S. Santini, R. Siano and D. Bass: Vampiers in the oceans: Predatory cercozoan amoebae in marine habitats. The ISME J., 7, 2387-2399 (2013).

Blinova, L., A. Bartosova and K. Gerulova: Cultivation of Microalgae (Chlorella vulgaris) for biodiesel production. J. Slovak Univ. Tech., 23, 87-95 (2015).

Carney, L.T. and T.W. Lane: Parasites in algae mass culture. Frontiers in Microbio., 5, 1-8 (2014).

Chiu, M.C., M.H. Kuo, H.Y. Chang and H.J. Lin: Bayesian modeling of the effect of extreme flooding and grazer community on algal biomass dynamics in a monsoonal Taiwan stream. Microb. Ecol., 72, 372380 (2016).

Day, J.G., N.J. Thomas, U.E.M. Achilles-Day and R.J.G. Leakey: Early detection of protozoan grazers in algal biofuel cultures. Bioresour. Technol., 114, 715-719 (2012).

Duerr, E.O., M.R. Edralin and N.M. Price: Facilities requirements and procedures for the laboratory and outdoor raceway culture of Spirulina spp. J. Mar. Biotechnol., 5, 1-11 (1997).

Felsenstein, J.: Confidence limits on phylogenies: An approach using the bootstrap. Evolution, 39, 783-791 (1985).

Gachon, C.M.M., M. Strittmatter, D.G. Muller, J. Kleinteich and F.C. Kuppler: Detection of differential host susceptibility to the marine Oomycete pathogen Eurychasma dicksonii by real time PCR: Not all algae are equal. Appl. Environ. Microbiol., 75, 322-328 (2009).

Gong, Y., D.J. Patterson, Y. Li, Z. Hu, M. Sommerfeld, Y. Chen and Q. Hu: Vernalophrys algivore gen. nov., sp. nov. (Rhizaria: Cercozoa: Vampyrellida), a new algal predator isolated from outdoor mass cultures of Scenedesmus dimorphus. App. Environ. Microbiol., 81, 3900-3913 (2015).

Han, F., J. Huang, Y. Li, W. Wang, M. Wan, G. Shen and J. Wang: Enhanced lipid productivity of Chlorella pyrenoidosa through the culture strategy of semi-continuous cultivation with nitrogen limitation and $\mathrm{pH}$ control by $\mathrm{CO}_{2}$. Bioresour. Technol., 136, 418-424 (2013).

Ho, S.S.T. and M. Alexander: The feeding of amebae on algae in culture. J. Phycology, 10, 95-100 (1974).

Ibrahim, M.A., M.S.M. Yusof and N.M. Amin: Anti-amoebic properties of carbonyl thiourea derivatives. Molecules, 19, 5191-5204 (2014).

Imaizumi, Y., N. Nagao, F.M. Yusoff, S. Taguchi and T. Toda: Estimation of optimum specific light intensity per cell on a high-cell-density continuous culture of Chlorella zofingiensis not limited by nutrients or $\mathrm{CO}_{2}$. Bioresour. Technol., 162, 53-59 (2014).

Jonckheere, J.F.D.: Isolation and molecular identification of Vahlkampfiid amoebae from an island (Tenerife, Spain). Acta Protozool., 45, 9196 (2006).

Karpov, S.A., K.V. Mikhailov, G.S. Mirzaeva, I.M. Mirabdullaev, K.A. Mamkaeva, N.N. Titova and V.V. Aleoshin: Obligatory phagotrophic aphelids turned out to branch with the earliest-diverging fungi. Protist., 164, 195-205(2013).

Letcher, P.M., S. Lopez, R. Schmieder, P.A. Lee, C. Behnke, M.J. Powell and R.C. McBride: Characterization of Amoeboaphelidium 
protococcarum, an algal parasite new to the Cryptomycota isolated from an outdoor algal pond used for the production of Biofuel. PLOS ONE, 8, e56232 (2013).

Lincoln, E.W, T.W. Hall and B. Koopman: Zooplankton control in mass algal cultures. Aquaculture, 32, 331-337 (1983).

Ma, A.T., J. Beld and B. Brahamsha: An amoebal grazer of cyanobacteria requires cobalamin produced by heterotrophic bacteria. Appl. Environ. Microbiol., 83, e00035-17 (2017).

Mahan, K.M., O.W. Odom and D.L. Herrin: Controlling fungal contamination in Chlamydomonas reinhardtii cultures. BioTechniques, 39, 457-458 (2005).

Mendes, L.B.B. and A.B. Vermelho: Allelopathy as a potential stretgy to improve microalgal cultivation. Biotechnol. Biofuels, 6, 1-14 (2013).

Mendez, C. and E. Uribe: Control of Branchionus sp. and Amoeba sp. in cultures of Arthrospira sp. Lat. Am. J.Aquat. Res., 40, 553-561 (2012).

Panova, T.G.T and J.G. Ivanova: Interactions between the unicellular red alga Rhodella reticulata (Rhodophyta) and contaminated bacteria. J. Appl. Microbiol., 93, 497-504 (2002).

Pipes, W.O: Carbondioxide limited growth of Chlorella in continuous culture. Appl. Microbiol., 10, 281-288 (1962).

Richmond, A. and E.W. Becker: Technological aspects of mass cultivation, a general outline, Handbook of microalgal mass culture. CRC Press, Boca Raton, Florida, $1^{\text {st }}$ Edn., USA (1986).

Ristaino, J.B., M. Madritch, C.L. Trout and G. Parra: PCR amplification of ribosomal DNA for species identification in the plant pathogen genus phytophthora. App. and Env. Microbiol., 64, 948-954 (1998).

Robles, A.G., L.S. Villatoro, M.G. Lazaro, M.O. Molina and A.M. Palomo: Vahlkampfia sp: Structural observations of cultured trophozoites. Exp. Parasitol., 130, 86-90 (2012).

Saitou, N. and M. Nei: The neighbor joining method: A new method for reconstructing phylogenetic trees. Mol. Bio. and Evol., 4, 406-425 (1987).

Scherholz, M.L. and W.R. Curtis: Achieving pH control in microalgal cultures through fed-batch addition of stoichiometrically-balanced growth media. BMC Biotech., 13, 1-16 (2013).

Smirnov, A.V. and T. Fenchel: Vahlkampfia anaerobica n. sp. and Vannella peregrinia $\mathrm{n}$. sp. (Rhizopoda) Anaerobic amoebae from a marine sediment. Arch. Protistenk., 147, 189-198 (1996).

Zhang, C.D., L. Wei, Y.H. Shi, Y.G. Li, J.K. Huang and H.X. Li: A new technology of $\mathrm{CO}_{2}$ supplementary for microalgae cultivation on large scale- A spraying absorption tower coupled with an outdoor open runway pond. Bioresour. Technol., 209, 351-359 (2016).

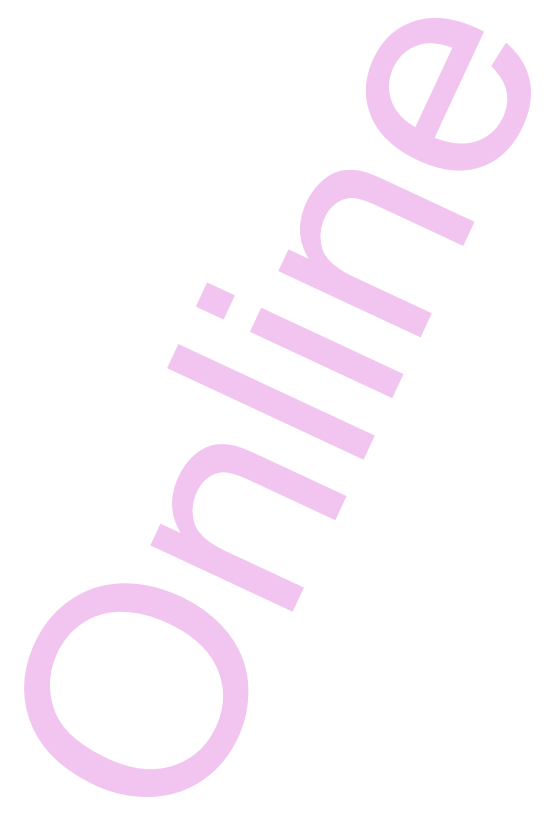

* Journal of Environmental Biology, January 2018 